\title{
A Case Report: Methotrexate and Etoposide as Adjuvant Therapy on Patient with Hydatidiform Moles that Have Growing Rapidly Becomes Gestational Trophoblastic Neoplasia
}

\author{
Supriyono
}

\section{ABSTRACT}

\begin{abstract}
A hydatidiform mole is a type of placental abnormality that is associated with gestational trophoblastic disease (GTD). It can be complete or partial mole. They are typically considered benign, but they are also premalignant, with the potential to become malignant and invasive to other organs (gestational trophoblastic neoplasia/GTN). Management of the hydatiform moles is curettage therapy and suction, which aims to therapy and diagnostic. Follow-up post evacuation of moles must be tight with serum hCG and clinical examination. When there is evidence of vaginal bleeding and an increase in serum hCG, chemotherapy must be used as an adjuvant. This case report intends to persuade clinicians that adjuvant therapy with chemotherapy combination-based Methotrexate/MTX and etoposide can recover and eliminate vaginal bleeding disorders. On her first pregnancy, a woman of $\mathbf{2 7}$ years old develops a complete hydatiform mole. The curettage and suction procedure were then performed twice. She also receives chemotherapy in three series of single MTX and three series of MTX plus Etoposide. Following this therapy, the patient recovers, normal hCG level and has no vaginal bleeding problems.
\end{abstract}

Keywords: Etoposide, hCG, hydatiform mole, gestational trophoblastic neoplasia/GTN, methotrexate.

\section{INTRODUCTION}

The most frequently reported GTD is hydatidiform mole (HM), a type of abnormal human pregnancy[1]. There are two main types of HM: benign and malignant. Invasion is a feature of the malignant type. According to the severity of histopathological alterations, karyotype variation, and the existence of embryonic elements, benign hydatid-form moles are classified as complete or incomplete or partial [2], [3]. $\mathrm{HM}$ is a benign form, but it can be classified as premalignant because it has the potential to become malignant, metastasize and common cause of gestational trophoblastic neoplasia (GTN) [4].

Geographic distribution affects the prevalence of GTN. Taiwan had the highest reported incidence of 1/125 live births, while Japan and Southeast Asia had 2/1000 pregnancies, the United States had 1/1500, and Europe had $1 / 1000$ [5]. It is widely estimated that the prevalence is highest in developing countries. The incidence has increased in women under the age of 20 and over the age of 40 . It is indeed greater in nulliparous women, a low-income country, as well as female patients whose diets are lacking in carotene, protein, and folic acid [6], uterine size up to 20 weeks, raising
Submitted : October 23, 2021

Published : January 7, 2022

ISSN: 2593-8339

DOI: $10.24018 /$ ejmed.2022.4.1.1116

\section{Supriyono*}

Department of Obstetry and Gynecology RSPAL Dr. Ramelan Surabaya - Faculty of Medicine, Universitas Hang Tuah, Surabaya, Indonesia.

(e-mail: supriyono.drspog@gmail.com)

*Corresponding Author
hCG levels above $100.000 \mathrm{mIU} / \mathrm{mL}$, lutein cysts' presence, medical history of second pregnancy with mole, post evacuation hemorrhage, the existence of lungs involvement, and medical issues of preeclampsia, histopathological characteristics of excessive trophoblastic hyperplasia, and histopathological characteristics of intense trophoblastic hyperplasia [7].

After treatment, most patients with hydatidiform moles will be cured. Even though about 15-20 percent of them will develop into GTN, which are malignant tumors [7]. Complete hydatiform moles (15-20\%) are more at risk of developing into neoplasms (GTN) than partial moles (less than 5\%) [6].

In most cases, HM are discovered during the first three months of pregnancy. Abnormality of vaginal bleeding was the most common clinical manifestation. Other clinical signs involve severe morning sickness/ hyperemesis gravidarum, an enlarged uterus; no fetus was found clinically or radiologically, pregnancy-induced hypertension, and abnormally high human chorionic gonadotropin or hCG levels [6], [8]. Complete mole's patients usually showed elevated serum hCG levels more than partial mole's patients. The biologic tumor marker of GTD is hCG, a hormone released by the placenta in all types of pregnancy [9]. Serial testing of hCG qualitative serum levels has several 
advantages, including to make a diagnosis HM, to assess post molar follow-up, to estimate cases which will go into spontaneous remission or, on the opposite, develop GTN, to control response to chemotherapy and surgical intervention, and to identify relapse situations [10].

Patients with hydatidiform moles are currently managed by improving general health, removing molar tissue, preventing malignancy, and detecting malignancy early after mole removal [7]. Suction curettage evacuation should be used to remove a hydatidiform mole, whereas suspected partial molar pregnancy may necessitate a combination of surgical management because the fetus's components can obstruct suction evacuation. Furthermore, hysterectomy remains a better option for appropriate surgical candidates who do not want to have another child in the future, as well as for older women who are at risk of contracting malignant adverse outcomes [1], [10]. Following molar evacuation, sequential serum hCG test should be performed utilizing commercially available laboratory tests designed to detect all forms of hCG to threshold levels. (Can detect hCG level $<5$ milliinternational units $/ \mathrm{mL}$ ). Preferably, hCG levels should be measured within two days after molar removal and then monitored regularly every one until two weeks when hCG levels begin to escalate [9].

The International Federation of Gynecology and Obstetrics or FIGO 2000 guidelines define the diagnostic criteria for GTN as follows:

1. Persistent hCG levels after four or more laboratory testing in three weeks,

2. an increase in hCG concentration measured three times in a row for at least two weeks,

3. choriocarcinoma was identified histologically,

4. an increase in the concentration levels of hCG for six months or more [11], [12].

GTN prophylactic therapy was carried out based on high risk or low risk classification based on FIGO criteria 2000. Patients classified as low-risk GTN receive single chemotherapy while high-risk patients receive combination chemotherapy. The several regimens of chemotherapy that are commonly used for GTN treatment including MTX, actinomycin D, vincristine, cyclophosphamide, and etoposide.

\section{CASE REPORT}

A 27-year woman G1P0A0, $10^{\text {th }}$ weeks of gestational age, came to the hospital, with a primary complaint of vaginal blood loss accompanied by clots formation for a few hours before consultation Her symptoms included abdominal pain, nausea, vomiting, and a loss of appetite. She had just two antenatal care examinations with a midwife without any ultrasound test. Based on physical examination: the patient was alert, she had normal pulse rate (97 BPM), blood pressure was $125 / 85 \mathrm{mmHg}$, her respiration rate was around 18 breaths per minute, and the temperature was $36.3^{\circ} \mathrm{C}$. There was no exophthalmos or thyroid gland enlargement. The cardiology assessment confirmed no abnormality of cardiac sounds such as murmurs or gallop. The abdomen palpation was soft, with tenderness, and a palpably enlarged uterus at 16 weeks.

The doctor found the vulva and vagina to be normal during the obstetrics and gynecology examination. The vaginal canal appeared smooth on speculum examination, as did the cervix, and there was minimal blood from the ostium of the cervix; no mass was visible. The uterus was palpated enlarged to the size of the 16th week of pregnancy during a bimanual examination, with no tenderness or adnexal mass palpated.

The following ultrasound discovered an enlarged endometrial cavity containing a massive, heterogeneous solid mass with multiple hypoechoic cysts, without any embryo indicating complete molar pregnancy; there's no mass on the adnexa or free fluid on the rectouterine pouch; and the bilateral ovarium was normal. According to laboratory results showed $\mathrm{Hb}=10.2 \mathrm{~g} / \mathrm{dl}, \mathrm{Ht}=32 \%$, platelets $=229.000 / \mathrm{ul}$, leukocytes $=9.400 / \mathrm{ul}$, based on the laboratory result, the patient has an anemia condition (decreasing $\mathrm{Hb}$ and $\mathrm{Ht}$ ).

The doctor determined that the patient would undergo suction curettage twice (first curettage at Mei 182018 and second curettage at Juni 1 2018) and followed by histopathological examination. The results of histopathological examination confirmed that the patient had a complete hydatidiform mole. After both curettage evacuation therapy, the patient still complained of vaginal bleeding, abdominal pain, and serial high hCG levels above $1000 \mathrm{mIU} / \mathrm{ml}$ for 14 days (days 1,7 and 14), so the doctor decided to refer the patient to Dr Ramelan Naval HospitalSurabaya for further examination and treatment at Juli 19 2018.

At Dr Ramelan Naval hospital, the hCG test was repeated and showed that the hCG level was still high at $2479 \mathrm{mIU} / \mathrm{ml}$, so the doctor decided to give chemotherapy single MTX. A thorough examination (X-ray and CT scan) was conducted before the chemotherapy administration to determine whether the patient was classified as having low-risk or high-risk GTN. The International Federation of Gynecology and Obstetrics/FIGO staging recommendations and the WHO predictive scoring system are used to categorize patients. [12]-[14].

1. FIGO staging recommendations consists of four stages. The uterine corpus is the only location where a neoplasmic mass can be found.

2. GTN extended to other parts of genital structures (vagina, adnexa, etc.).

3. GTN is accompanied by lung metastases, with or without the involvement of the genital systems.

4. GTN is accompanied by distant metastasis to other organs [11].

The WHO prognostic scoring system is composed of eight factors that influence the prognosis or progression of GTN., including age, previous pregnancy, the time between the previous pregnancy and the initiate of chemotherapy, level of hCG previous to chemotherapy regime, Diameter of the tumor, presence of metastatic disease, total number of metastatic disease, and medical history of chemotherapeutic agents malfunction [12]-[14]. One of the following conditions was used to determine low-risk disease: FIGO scoring system stage I, or stage II, or stage III accompany with a WHO predictive risk score of less than seven. One of the following requirements, on the other hand, defined high-risk classification:

1. FIGO scoring system stage four or

2. FIGO scoring system stages two or three accompany with a WHO predictive risk score greater than six [13]. 
Based on FIGO and WHO scoring assessments, the patient has low risk of GTN (FIGO 1 with WHO risk score 5).

The patient received single chemotherapy, three series of MTX regimen for 5 days at a dose of $0.3 \mathrm{mg} / \mathrm{kg}$ of BW intravenously, followed by a 2-week break [13]. After administration of 3 series of MTX, the hCG level was still high above $1000 \mathrm{mIU} / \mathrm{ml}$, and the patient was still experiencing vaginal bleeding. The doctor decided to conduct three series round of combination chemotherapy of MTX and etoposide for five days. MTX at a dose of $0.3 \mathrm{mg} / \mathrm{Kg}$ of BW intravenously [13] with intravenously administered 100 $\mathrm{mg} / \mathrm{m}^{2}$ etoposide (infusion over 30 minutes) [14]. One weeks after administration of three series of MTX and etoposide combination, an examination of hCG levels revealed a significant decrease to $27.2 \mathrm{mIU} / \mathrm{ml}$. Monitoring in the fourth and sixth week after administration of combination chemotherapy revealed that the hCG level was less than five $\mathrm{mIU} / \mathrm{ml}(2 \mathrm{mIU} / \mathrm{ml}$, it means hCG levels were less than the baseline of normal hCG level); the patient had no vaginal bleeding, no abdominal pain, and ultrasound results revealed a normal picture. The patient was declared cured and instructed to use contraception to postpone pregnancy for up to a year, as well as to take vitamin A every day to avoid a molar pregnancy.

\section{DISCUSSION}

The patient has a complete hydatidiform/CM mole, which can occur because of abnormal fertilization. $\mathrm{CM}$ have a karyotype of 46, XX, with paternal origin for all chromosomes, lack maternal chromosomes [15]. About $15 \%$ to $20 \%$ of the female with a complete hydatidiform mole will generate post molar GTN, while only $0.5 \%$ to $5 \%$ of the female with a partial hydatidiform mole would then develop malignant lesions [14].

In molar pregnancy, there are several treatment options available, including suction curettage, and hysterectomy [1], [10]. Because the patient is still of reproductive years, so she could not have a hysterectomy, the doctor decided to perform curettage and suction on her. Suction curettage approach is preferred by the majority of patients who want to preserve their reproductive role, which is typically conducted under ultrasound assistance [9]. The patient's hCG level remained high, with symptoms of vaginal bleeding and abdominal pain still predominating, after twice suction curettage administration. The doctor diagnosed the patient with postmolar pregnancy GTN based on the progression of the patient's manifestation, so the doctor performed FIGO scoring and WHO prognosis assessment to determine therapy for the patient. According to the scoring evaluations, the patient is in the low-risk group and should receive single chemotherapy, MTX.

GTN is a neoplasm that easily metastasizes and has a high mortality rate prior to the discovery of chemotherapy. However, the discovery of MTX at 1950, antineoplastic regiment that is effective in minimizing the development of GTN, can reduce mortality and increase the complete remission for GTN without any metastatic manifestation [12]. Advances in the pharmaceutical field have also increased the cure rate for GTN with metastases through the use of a drug combination (cisplatin or etoposide) [16].
After a single chemotherapy administration of MTX, or etoposide (ETP), the low-risk GTN achieves nearly 100 percent complete remission patients [17]. Methotrexate can effectively inhibit trophoblast cell proliferation by inhibiting DNA replication and the cell cycle in the S phase [18]. Because of its pharmacological efficacy, overall safety, toxicity profile, longevity, and cost-effectiveness, MTX is extensively used in medical practice. Administration of MTX could be accompanied by folic acid to minimize its toxicity, but it can be given a single regimen without folic acid. There are differences in MTX doses, durations, and methods of administration, but a meta-analysis study has proved that intravenously administered MTX for 5 days was the most effective regimen for achieving complete remission in lowrisk GTN cases [13].

However, approximately $20 \%$ until $30 \%$ of GTN patients receiving chemotherapy must alter their regimens because of drug resistance, severe side effect and relapse [17], [19] . Age, FIGO stage and score, prechemotherapy - hCG level, and the nature of the previous pregnancy were all found to be associated with drug resistance in univariate analysis [19]. Another study discovered that a higher WHO scoring system (5-6) and choriocarcinoma diagnosis are both linked to an increased risk of resistance to single agent chemotherapy. It is reasonable to consider reducing the cutoff point for using multiple agent chemotherapy in these otherwise low-risk patients [14]. In this study, the WHO scoring prognosis of the patient was 5; this could be one of the factors resulting the inadequacy of MTX single chemotherapy. Another possibility that caused the failure of MTX therapy in this case, was the short period between the diagnosis of CM and GTN (less than two month, marked by persistently high hCG levels and vaginal bleeding that occurred continuously after the patient's molar tissue was removed). Study of Ngan et al (2018) showed that almost all the cases of GTN manifest within 6-12 months after molar evacuation. The probability of having post molar GTN is extremely low after hCG normalization has been confirmed with a second hCG value [9].

Etoposide/ ETP has been widely used as chemotherapy of a variety of neoplasms in the United States, including breast cancer, lung cancer, testicular cancer, and other cancers. ETP works by inhibiting the topoisomerase II enzyme, causing the DNA double chain to break, and preventing it from religating. ETP binds specifically to chromosomes and histones, allowing them to inhibit mitosis and stop cell division in the G2 and S phases. Through activation of the caspase 9 pathway, ETP can induce cell apoptosis. ETP has a more complex activity to inhibit the growth of cancer cells than MTX based on its mechanism of action [20]. According to the case report, the patient achieved complete remission after only one series of MTX and ETP for 5 days. The success of this combination therapy is due to the two drugs' complementary actions. Kanno et al (2018) reported the same result, that single MTX administration to low risk GTN patients resulted in remission failure (35\%). To overcome this condition, a second line of therapy is required; one of the combinations used is MTX and ETP for 5 days, and complete remission is achieved by 83.3 percent of the total patients who experience therapy failure using single chemotherapy. According to these study's findings, if hCG titers remain 
stable or keep rising during 1st line chemotherapy drug, or if the patients does not respond following the second series of the same drug administration for any reason, combination chemotherapy with two different types of drugs could be considered [17].

\section{CONCLUSION}

The use of FIGO scoring and WHO prognostic scoring assists clinicians in determining the chemotherapy used in GTN patients; however, a borderline low-risk score (WHO score 5-6) should be considered for the use of combination chemotherapy. The level of hCG that persists or increases after the administration of the first/second regimen, and the distance between mole evacuation and the appearance of GTN that is less than six months, are two indicators that could be used as a reference for changing single to combination chemotherapy in low-risk GTN patient.

\section{ACKNOWLEDGMENT}

There is no conflict of interest in the publication of the paper. The author would like to thank all stakeholders who assisted in the preparation of this case report, particularly Siti Khotijah Hospital and Dr.Ramelan Naval HospitalSurabaya.

\section{REFERENCES}

[1] Farah G, Nainggolan JD. The Controversy of Hydatidiform Mole Treatment In Women age $\geq 40$ year-old. Medicinus. 2018; 6(2): 511.

[2] Hosseini S, Varazgaei MY, Ebrahimi M. Effect of different doses of vitamin a on $\beta$-hcg production in patients suffering from gestational trophoblastic disease. J. Adv. Med. Biomed. Res. 2020; 28(128): 151-155.

[3] Lund H, Vyberg M, Eriksen HH, Grove A, Jensen AØ, Sunde L. Decreasing incidence of registered hydatidiform moles in Denmark 1999-2014. Sci. Rep. 2020; 10(1): 1-10.

[4] Kalogiannidis I, Kalinderi K, Kalinderis M, Miliaras D, Tarlatzis B, Athanasiadis A. Recurrent complete hydatidiform mole: where we are, is there a safe gestational horizon? Opinion and minireview. J. Assist. Reprod. Genet. 2018; 35(6): 967-973.

[5] Zakaria A, Hemida R, Elrefaie W, Refaie E. Incidence and outcome of gestational trophoblastic disease in lower Egypt. Afr. Health Sci. 2020; 20(1): 73-82.

[6] Mulisya O, Roberts DJ, Sengupta ES, Agaba E, Laffita D, Tobias $\mathrm{T}$, et al. Prevalence and Factors Associated with Hydatidiform Mole among Patients Undergoing Uterine Evacuation at Mbarara Regional Referral Hospital. Obstet. Gynecol. Int. 2018; 2018.

[7] Tobing M, Arabia F, Hidayat Y, Mantilidewi K. Relationship of histopathological representation of excessive proliferation of trophoblast cells with the possibility of malignant events after complete hydatidiform mole. Int. J. Gen. Med. 2021; 14: 18991904.

[8] Hidayat R, Riviati N. Sarcopenia from Pathophysiology to Clinical : Literature Review. Biomed. J. Indones. 2021; 7(1): 125140.

[9] Soper JT. Gestational Trophoblastic Disease: Current Evaluation and Management. Obstet. Gynecol. 2021; 137(2): 355-370.

[10] Anyanwu M, Bah K. A cross sectional descriptive study on hydatidiform mole at Gambian tertiary hospital. Women's Heal. 2020; 9(1): 1-5.

[11] May T, Goldstein DP, Berkowitz RS. Current Chemotherapeutic Management of Patients with Gestational Trophoblastic Neoplasia. Chemother. Res. Pract. 2011; 2011: 1-12.

[12] Braga A, Mora P, de Melo AC, Nogueira-Rodrigues A, AmimJunior J, Rezende-Filho J, et al. Challenges in the diagnosis and treatment of gestational trophoblastic neoplasia worldwide. World J. Clin. Oncol. 2019; 10(2): 28-37.

[13] Alobaid A, Ahmeed S, Abuzaid M, Aldakhil L, Abu-Zaid A. Lowrisk gestational trophoblastic neoplasia: A single-center experience from Saudi Arabia. Avicenna J. Med. 2019; 9(3): 89-93.

[14] Ngan HYS, Seckl MJ, Berkowitz RS, Xiang Y, Golfier F, Sekharan PK, et al. Update on the diagnosis and management of gestational trophoblastic disease. Int. J. Gynecol. Obstet. 2018; 143: 79-85.

[15] Candelier JJ. The hydatidiform mole. Cell Adhes. Migr. 2016; 10: (1-2): 226-235.

[16] Berkowitz RS, Goldstein DP. Current advances in the management of gestational trophoblastic disease. Gynecol. Oncol. 2013; 128(1): $3-5$.

[17] Kanno T, Matsui H, Akizawa Y, Usui H, Shozu M. Treatment results of the second-line chemotherapy regimen for patients with low-risk gestational trophoblastic neoplasia treated with 5-day methotrexate and 5-day etoposide. J. Gynecol. Oncol. 2018; 29(6): 6-13.

[18] Koźmiński P, Halik PK, Chesori R, Gniazdowska E. Overview of dual-acting drug methotrexate in different neurological diseases, autoimmune pathologies and cancers. Int. J. Mol. Sci. 2020; 21(10).

[19] Qiu Q, Zheng X. Comparison of effects of methotrexate/calcium folinate and actinomycin D on trophoblastic tumors. All Life. 2020; 13(1): 569-576.

[20] Kluska M, Woźniak K. Natural polyphenols as modulators of etoposide anti-cancer activity. Int. J. Mol. Sci. 2021; 22(12).

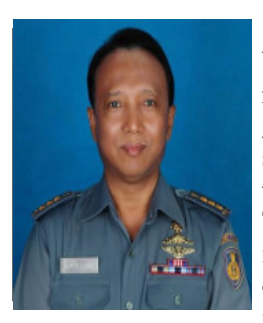

Supriyono, dr., SpOG(K) Onk, The author was born in Surabaya on June 21, 1954. He started his medical studies at Airlangga University and graduated in 1981. In 1992, he completed a specialist degree in obstetrics and gynecology at the Faculty of Medicine, Airlangga University. Then In 2000, he was obtained a consultant degree in oncology at the Faculty of Medicine, University of Indonesia. From 1981 to 2012, the author served in the Republic of Indonesia military. The author has been a lecturer at Hang Tuah University Medical Faculty since 2005 until now. The author is interested in conducting research on neoplasms in the fields of obstetrics and gynecology, epidemiology, pathophysiology, histopathology, therapy, and immunology. 